MATEC Web of Conferences 32, 01003 (2015)

DOI: $10.1051 /$ matecconf $/ 20153201003$

(C) Owned by the authors, published by EDP Sciences, 2015

\title{
MEMS acceleration sensor with remote optical readout for continuous power generator monitoring
}

\author{
Maurizio Tormen ${ }^{1, a}$, Branislav Timotijevic ${ }^{1}$, Yves Pétremand ${ }^{1}$, Markus Lützelschwab ${ }^{1}$, Dara Zaman Bayat ${ }^{1}$, Yvan Jacquat ${ }^{2}$, \\ Laurent $\mathrm{Aebi}^{2}$
}

${ }^{1}$ CSEM SA, Rue J. Droz 1, 2002 Neuchatel, Switzerland

${ }^{2}$ MC-Monitoring SA, Route André Piller 19, 1762 Givisiez, Switzerland

\begin{abstract}
Miniaturized accelerometers with remote optical readout are required devices for the continuous monitoring of vibrations inside power generators. In turbo and hydro generators, end-winding vibrations are present during operation causing in the long term undesirable out-of-service repairs. Continuous monitoring of these vibrations is therefore mandatory. The high electromagnetic fields in the generators impose the use of devices immune to electromagnetic interferences. In this paper a MEMS based accelerometer with remote optical readout is presented. Advantages of the proposed device are the use of a differential optical signal to reject the common mode signal and noise, the reduced number of steps for the MEMS chip fabrication and for the system assembly, and the reduced package volume.
\end{abstract}

\section{Introduction}

Miniaturized accelerometers with remote optical readout are required devices for the continuous monitoring of vibrations inside power generators. In turbo and hydro generators, end-winding vibrations are present during operation at twice the electrical synchronous frequency of the generator. High vibrations can lead to loosening of entire end-winding support system, wear of insulation material, rupture of coil and fatigue cracking of conductor, all of which require out-ofservice repairs. Continuous monitoring of these vibrations is therefore mandatory and the high electromagnetic fields in the generators impose the use of devices by design non-conductive and immune to electromagnetic interferences.

Existing solutions in the market are based on manual assembly of a relevant number of micro-machined and micro-optical elements, often implying limited frequency range available for monitoring due to relatively high masses and, in terms of production, high manufacturing costs and low yields.

A Fiber-optic Acceleration Sensor (FAS) is by design non-conductive and immune to electromagnetic interferences. Its passive principle makes it ideal for shock and vibration measurements in areas where conventional accelerometers (e.g. piezoelectric or MEMS with metal wiring) may create electrical discharges, cause changes in magnetic field distribution, prove hazardous to personnel, and impair reliable operation. FAS sensors are therefore an optimal solution to monitor end-winding vibration during generator operation.

\footnotetext{
${ }^{a}$ Corresponding author: maurizio.tormen@esem.ch
}

FAS sensors are used as well in numerous other applications including rotating machinery, high voltage transformers, circuit breakers, high voltage electrical transmission lines, explosive atmospheres, etc. System monitoring leads to operating costs advantages, such as optimal planning of maintenance tasks, reduction of unplanned downtime, increase of the machine availability.

A number of different approaches to FAS have been explored: a general overview can be found in [1]. Recent works have mainly explored solutions based on Grating filters ([2, 3], Fabry-Perot filters [4], Michelson interferometer [5].

In this paper a MEMS based accelerometer with remote optical readout is presented. Advantages compared to state of the art devices for such application are a simplified electronics when compared to wavelength or phase based measurement systems, high rejection of the common mode signal and high measurement linearity thanks to a differential intensity signal approach, low cost MEMS chip given the reduced number of fabrication steps and the number of chips per wafer (more than 400 on a 6" wafer), low cost and high yield system assembly thanks to a simple and robust assembly process, low barriers to market-entry since the reduced package volume is compatible with the already allocated space in turbo generators. For electromagnetic immunity, no metal is present in the sensor head.

\section{Opto-mechanical design}

The MEMS chip is composed of a seismic mass,

This is an Open Access article distributed under the terms of the Creative Commons Attribution License 4.0, which permits unrestricted use, distribution, and reproduction in any medium, provided the original work is properly cited. 
suspended through springs, with opened regions to introduce a damping gel, of a 2-facet mirror, moving with the seismic mass, redirecting the optical signal from an input fiber into two output fibers. In order to reduce the system dimensions the two output fibers were not placed at $90 \mathrm{deg}$ in respect to the input fiber as in [1], but at a shallower angle, $70 \mathrm{deg}$ or $45 \mathrm{deg}$. Figure 1 reports the layout of the basic chip, with the input fiber and the two output fibers.

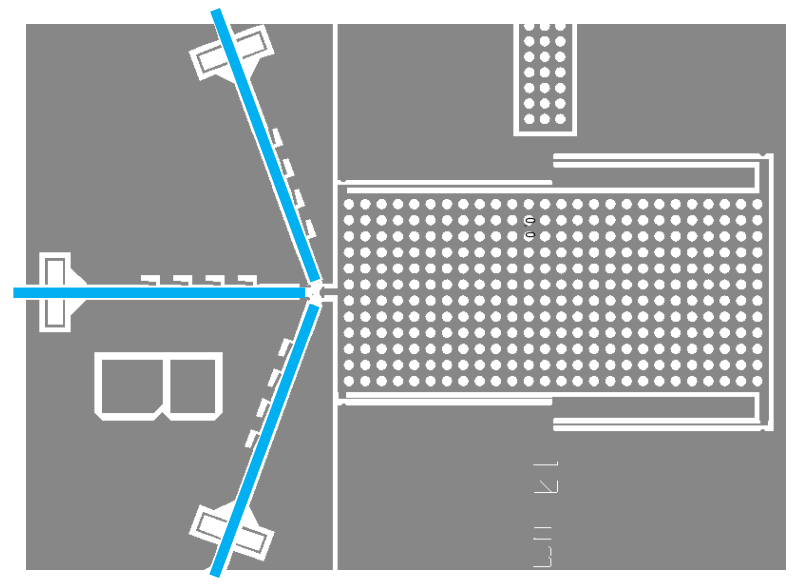

Figure 1. MEMS optical accelerometer layout.

The seismic mass and the springs were designed in order to provide sufficiently high resonance frequencies for the specific application: one family has the first resonance at $1 \mathrm{kHz}$, the second at $3 \mathrm{kHz}$ (Table 1). The lateral displacement of the mirror was design to be $10 \mu \mathrm{m}$ and $1 \mu \mathrm{m}$, respectively, at an acceleration of $40 \mathrm{~g}$.

Table 1 List of the investigated FAS family designs.

\begin{tabular}{|l|c|c|}
\hline & $\begin{array}{c}\text { First resonance } \\
\text { frequency }[\mathrm{Hz}]\end{array}$ & $\begin{array}{c}\text { Mass displacement }[\mu \mathrm{m}] \\
\text { at 40g }\end{array}$ \\
\hline Design 1 & 1'000 & 10 \\
\hline Design 2 & 3'000 & 1 \\
\hline
\end{tabular}

In absence of acceleration the signals in the output fibers are nominally identical (Figure 2, on the left); when the seismic mass is subjected to acceleration, the lateral displacement of the mirror induces an increase of optical power in one fiber and a reduction in the second one (Figure 2, on the right).
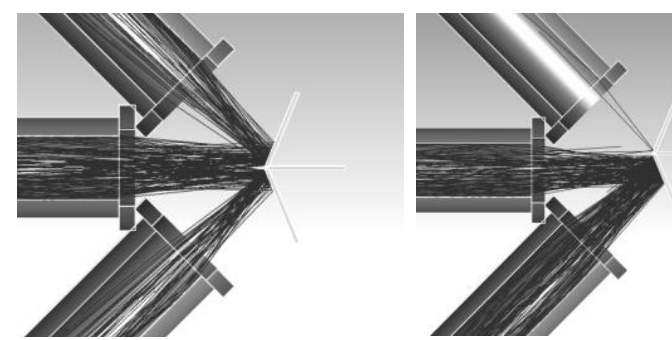

Figure 2. Ray-tracing simulation of the optical signal arriving from the input fiber (central one) and redirected by the moving mirror into the 2 output fibers (external ones) in absence (left) and presence (right) of acceleration.

The signals from the two output fibers are collected separately in remote (in Figure 3 left, the simulated power expected at the two output fibers ends, as a function of the lateral displacement of the mirror). The acceleration measurement is derived from the difference of the two signals (in Figure 3 right). Deviation from linearity is expected to be less than $1 \%$ for displacements up to $10 \mu \mathrm{m}$ for accelerations up to $40 \mathrm{~g}$. Analytical simulations and Zemax ray-tracing were performed in order to optimize the optical signal as a function of geometrical parameters of the system.
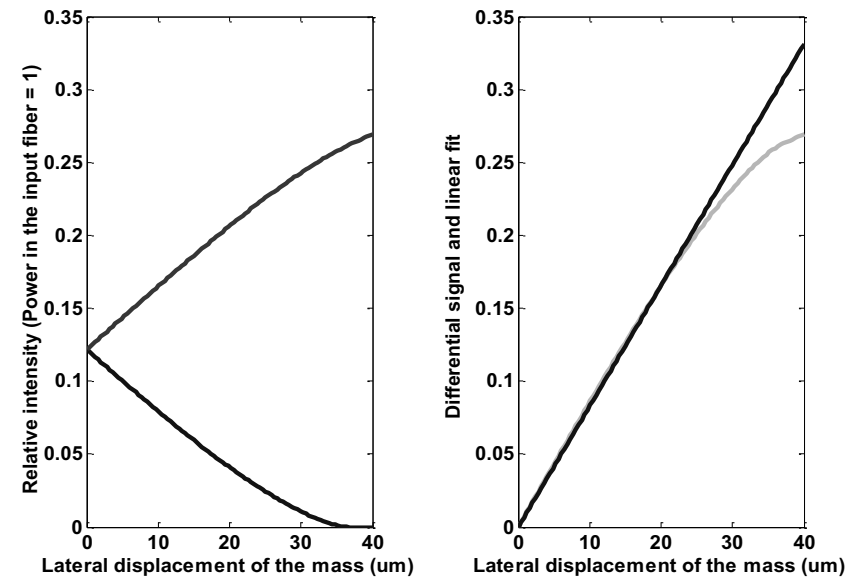

Figure 3. (Left) Simulation of the relative signals at the output fiber ends as a function of MEMS mirror lateral displacement. (Right) Differential signal at the two output fiber ends (gray line) and its linear fit (black line).

\section{Microfabrication process}

The microfabrication process (Figure 4) starts with 6" SOI (Silicon-On-Insulator) wafers with the following thicknesses: Handle layer $500 \mu \mathrm{m}$, buried oxide layer $3 \mu \mathrm{m}$ and device layer $120 \mu \mathrm{m}$. The process has been designed to have a single mask and DRIE step in order to reduce the process time and cost. The structures are patterned via photolithography with AZ 9260 photoresist. The obtained mask is transferred into silicon by DRIE etching through the entire device layer down to the buried oxide.

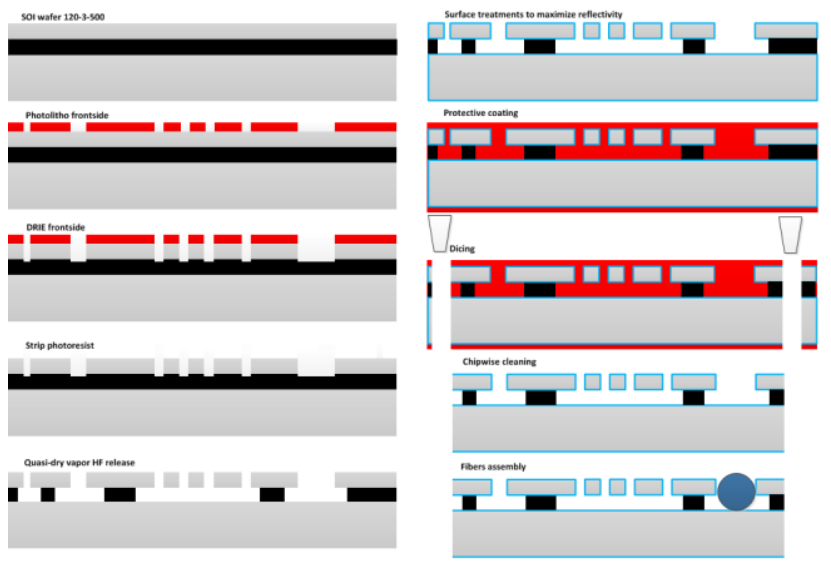

Figure 4. Schematic process flow of the MEMS optical accelerometer chip (cross-section view).

After stripping the remaining photoresist and cleaning the wafer, the buried oxide is partially etched to release the 
movable structures (Figure 5).

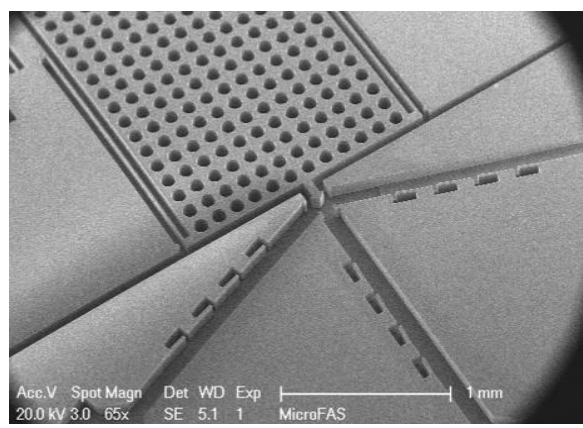

Figure 5. SEM picture of the fabricated device.

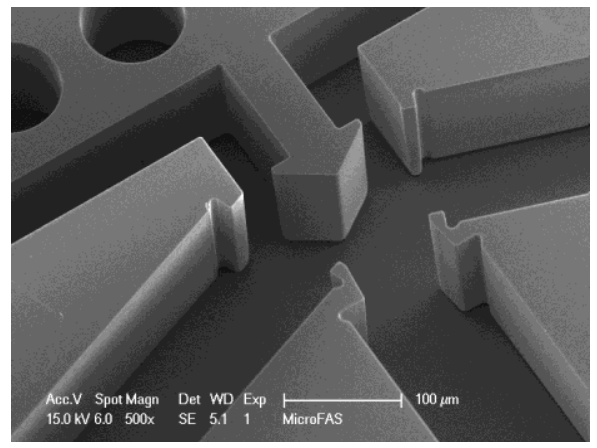

Figure 6. SEM image of the 2-facet movable mirror redirecting the optical signal and the U-grooves for optical fiber alignment.

The technique used is a timed quasi-dry etching by vapour HF. During this process step, it is necessary to sufficiently etch the oxide in order to release the movable structures, but not to overetch it, to maintain intact the oxide supporting the not-moving parts of the device layer. Surface treatments are introduced to polish the lateral surfaces in order to optimize the mirror reflectivity (Figure 6). A layer of photoresist is then poured on the surface of the wafer in order to protect the structures during dicing for singulation of the chips. The process continues at chip level with a final cleaning procedure to remove the protective photoresist: at this point the chip is ready for the assembly with the optical fibers in the package.

\section{Packaging}

The package provides a solid base for the interface between the MEMS device and the optical fibers. In terms of possible materials for the package, metal parts are excluded because of the strong electromagnetic fields present in the generators; moreover temperatures of up to $155^{\circ} \mathrm{C}$ and a hydrogen rich atmosphere (5bars) represent further constraints in the choice of the package material. Finally, hermetic package is not required for the applications. Based on this input, PEEK was chosen because of its physical and chemical properties as well as its adequate machinability.

Figure 7 (left) shows an assembled device. The optical fibers are completely molded in UV curable adhesive, thereby providing an effective strain relief. Without it, the fibers would exert a detrimental force on the MEMS chip during both the handling as well as operations. The output fibers are placed at an angle of $\alpha$ (70 deg or $45 \mathrm{deg}$ ) in respect to the central input fiber (Figure 7, right). The fiber alignment is performed using a six axis alignment stage (F-206 from PI) with an arbitrary pivot point. To facilitate the fiber insertion the MEMS chip hosts some fiber alignment features; on top of that a pre-tilting of the fiber during insertion was introduced to slide the fibers into their respective channels on the MEMS chip. The PEEK base features as well channels for the guidance of the three fibers. The outer two channels are curved with radii, r, so that the minimal bending radius of the fiber can be maintained during alignment and operation. The radius was chosen as small as possible to reduce the overall package size and at the same time reduce the bending losses to an acceptable level (0.3-0.4 dB).

For mechanical protection, a glass lid is added to the package at a final step. Figure 8 shows the fully assembled device with connectorized fibers for ready for the characterization.
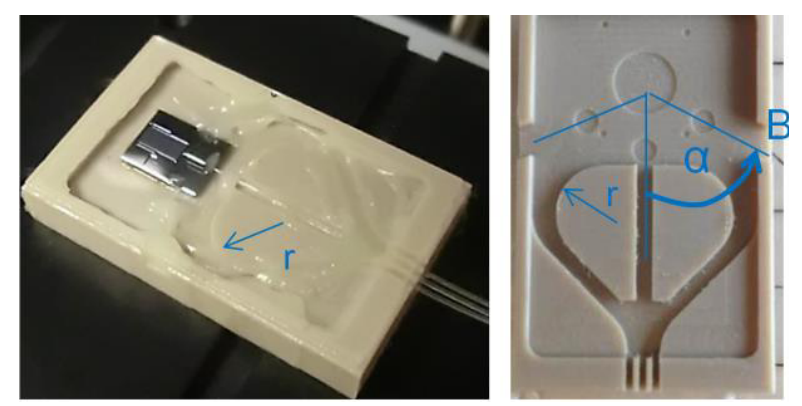

Figure 7. Left. Assembled device. Right: geometrical features of the PEEK package.

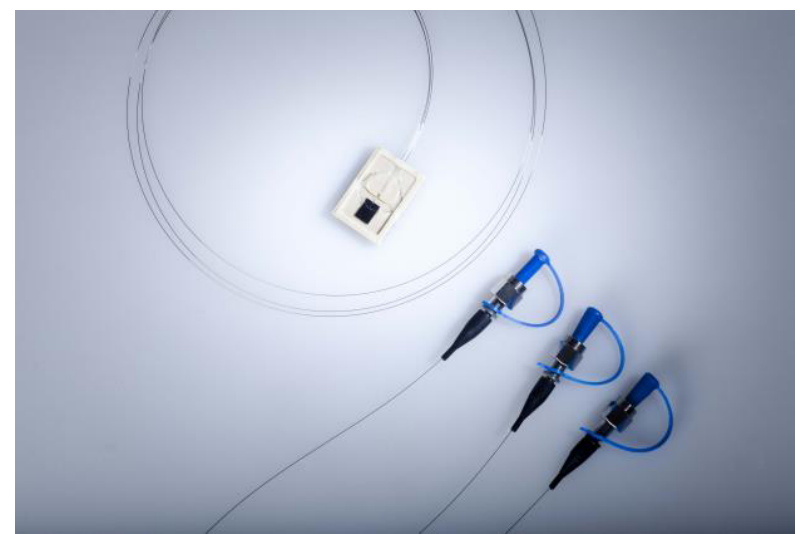

Figure 8. Fully assembled and connectorized optical accelerometer sensor.

\section{Characterization}

Table 2 reports the comparison between the simulated resonance frequencies and the ones experimentally obtained for the two designs presented in Table 1. Two different techniques were used to measure the resonance frequency. The first technique uses a shaker, a reference piezo sensor with its charge amplifier and a function generator to have a complete frequency response; the second technique makes the device vibrate at increasing frequencies with a piezo actuator and the resonance frequency is in correspondence with the maximum 
displacement of the seismic mass.

Table 2. Comparison of the resonant frequency obtained, numerically and experimentally.

\begin{tabular}{|l|c|c|c|}
\hline & $\begin{array}{c}\text { Simulated } \\
\text { resonance }[\mathrm{Hz}]\end{array}$ & $\begin{array}{c}\text { First } \\
\text { setup }\end{array}$ & $\begin{array}{c}\text { Second } \\
\text { setup }\end{array}$ \\
\hline Design 1 & 989 & 849 & 837 \\
\hline Design 2 & 3132 & 2674 & 2645 \\
\hline
\end{tabular}

A difference of $\sim 1 \%$ in measured resonance frequency is obtained between the two experimental methods. A more significant variation $(\sim 15 \%)$ is present between the numerical simulations and the experimental results. From an application point of view, despites the mentioned discrepancy, the obtained results for the resonance frequency are in line with the requirements.

The frequency response for two chips from the two different families is reported in Figure 9.

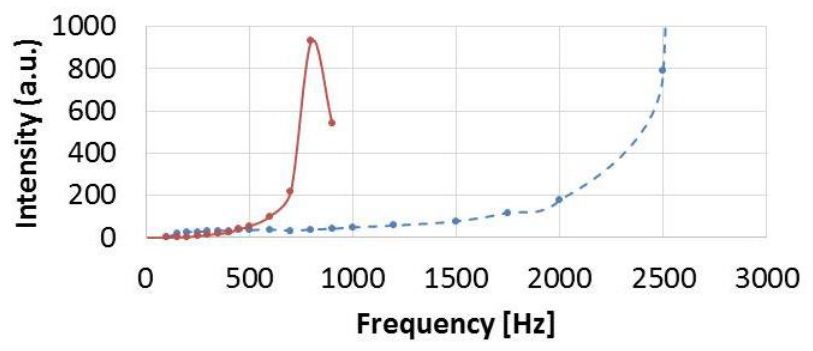

Figure 9. Frequency response of two chips from the two families presented in Table 1.

In order to flatten the frequency response in the frequency range of interest $(10 \mathrm{~Hz}-400 \mathrm{~Hz}$ for the first family, and $50 \mathrm{~Hz}-1500 \mathrm{~Hz}$ for the second family), the second generation of devices will include damping structures.

The dynamic range is reported in Figure 10 for the two families (continuous line for family 1, dashed line for family 2). Family 1 has a better dynamic range at lower frequency down to $0.2 \mathrm{~g}$. At $0.2 \mathrm{~g}$ the deviation from $7 \mathrm{~g}$ is still below 5\% while family 2 presents more than $30 \%$ deviation. Below $0.2 \mathrm{~g}$ the signal to noise ratio comes close 1.

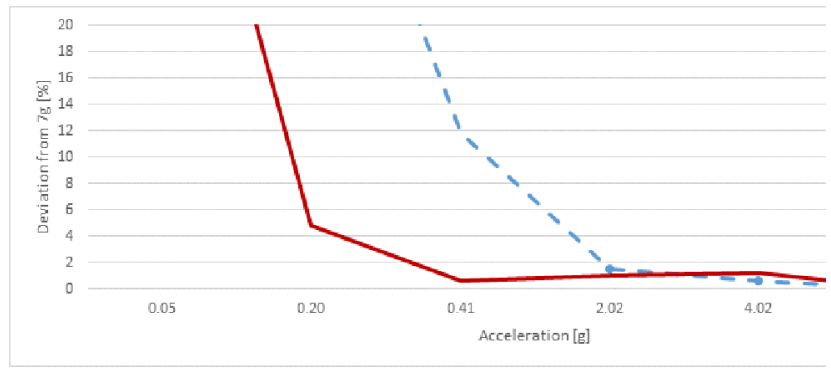

Figure 10. Dynamic range analysis of two chips from the two families presented in Table 1.

\section{Conclusions}

In the present paper a MEMS based accelerometer with remote optical readout is presented. First results showed high rejection of the common mode signal thanks to a differential intensity signal approach, low cost MEMS chip given the reduced number of fabrication steps and the number of chips per wafer, low cost and high yield system assembly thanks to a simple and robust assembly process.

Future work foresees the introduction of damping structures to improve the flatness of the frequency response in the frequency range of interest for the measurement.

\section{References}

1. B. Guldimann, "Micromachined fiber optic accelerometer based on intensity modulation", PhD thesis, University of Neuchate 1,2001 .

2. Chongyu Lin ; Hong Luo ; Shuidong Xiong and Haitao Li; "Investigation on a fiber optic accelerometer based on FBGFP interferometer ", Proc. SPIE 9297 , International Symposium on Optoelectronic Technology and Application 2014: Laser and Optical Measurement Technology; and Fiber Optic Sensors, 929735 (December 3, $2014)$;

3. Yeon-Gwan Lee et al, "Performance of a single reflective grating-based fiber optic accelerometer" 2012 Meas. Sci. Technol. 23045101.

4. Zhong-zhu Yang; Hong Luo and Shuidong

Xiong

" High sensitivity fiber optic accelerometer based on folding F-P cavity ", Proc. SPIE 8914 , International Symposium on Photoelectronic Detection and Imaging 2013 : Fiber Optic Sensors and Optical Coherence Tomography, 891411 (August 29, 2013 );

5. Feng Peng, Jun Yang, Bing Wu, Yonggui Yuan, Xingliang Li, Ai Zhou, and Libo Yuan, "Compact fiber optic accelerometer," Chin. Opt. Lett. 10, $011201-(2012)$ 